\title{
Self-Care Practices of Preparatory School Students with Type 1 Diabetes Mellitus in ELBehira Governorate
}

\author{
Naglaa Kamel Abduallah Hussein*, Samiha Hamdi Sayed Ramadan, Amal Yuosef Abd ElWahed \\ Community Health Nursing Department, Faculty of Nursing, Damanhour University, Egypt \\ *Corresponding author: nogakamel55@yahoo.com
}

Received August 17, 2018; Revised September 27, 2018; Accepted October 06, 2018

\begin{abstract}
Background: Type 1 diabetes (T1D) is more prevailing disease in adolescence. Intervening in self-care is crucial for disease management and to reduce the risk of diabetes-specific complications. The aim of the study was to assess self-care practices of preparatory school students with type 1 Diabetes Mellitus in EL Behira Governorate. Egypt. Subjects: A total number of 300 students constituted the subjects of the study. Research design: A descriptive research design was used. Setting: Data was gained from governmental preparatory schools in El-Behira governorate. Sampling students were chosen by multistage sampling technique. Tools: one tool was used for data collection, a structured interview schedule composed of three parts to collect data about socio-demographic characteristics, students' knowledge about TID, and assessment of students' self-care practices. Results: $53.3 \%$ of the studied diabetic preparatory school students have fair total self-care practices score while $29.7 \%$ of them have poor self-care practices. A significant difference was revealed between the studied diabetic students' total score of knowledge and self-care practices. $76.7 \%$ of the studied diabetic preparatory school students have poor total knowledge score. A significant difference was revealed between the studied diabetic students' total score of knowledge and self-care practices In addition, diabetic students' age, previous hospitalization due to hypo/hyperglycemic coma, duration of disease discovery and socioeconomic status were significantly varied among them in relation to their total knowledge and self-care practices scores. Moreover, diabetic students' gender, father's education, mothers' education, mother's working condition were significantly varied among them in relation to their total self-care practices score. Conclusion and recommendations: The results of the present study concluded that the highest percentage of the studied diabetic preparatory school students have either fair or poor self-care practices and poor total knowledge score, so it is recommended to develop hotlines, mass media campaigns for dissemination of accurate information about juvenile diabetes and establishing an accurate surveillance and documentation system of diabetic students for easy accessibility and provision of accurate information.
\end{abstract}

Keywords: practice, diabetes, self-care, school' students

Cite This Article: Naglaa Kamel Abduallah Hussein, Samiha Hamdi Sayed Ramadan, and Amal Yuosef Abd ElWahed, "Self-Care Practices of Preparatory School Students with Type 1 Diabetes Mellitus in ELBehira Governorate." American Journal of Nursing Research, vol. 6, no. 6 (2018): 407-417. doi: 10.12691/ajnr-6-6-8.

\section{Introduction}

Type 1 diabetes mellitus (T1DM) is a highly prevalent chronic disease and an important public health problem especially in childhood and adolescent's stages. Additionally, T1DM is considered one of the most common metabolic and endocrine diseases among adolescents. [1] According to the International Diabetes Federation (IDF-2015), the number of children aged 0 to 14 years with T1DM was 542, 000 worldwide. [2] In Egypt, there's lack of information concerning prevalence of T1DM among adolescents; since the latest incidence of T1DM in children aged 0 to 14 was 8/100,000 according to the IDF-2011, [3] while in adult there were 8.222.600 cases of diabetes with prevalence rate $15.1 \%$ (IFD 2017). [4]

Diabetes mellitus can affect physical growth and development and lead to damage, dysfunction, or failure of numerous organs especially eyes, kidneys, nerves, etc. [5] Childhood diabetes is quick in its onset presenting with the classic triad of symptoms: polyuria, polydipsia, and polyphagia. In spite of the hunger and increase dietary intake, the child be defeated his weight. The symptoms can seem insidiously, with fatigue, anorexia, nausea, lethargy, and weakness. [6]

Individual with T1DM needs a resource of exogenous insulin to sustain his life. [7] Without insulin, the patient can develop diabetic ketoacidosis; a life threatening condition leading to metabolic acidosis. [8]

Self-care practices are crucial for controlling blood glucose level. Studies propose that as the maximum amount as $95 \%$ of the self-care is typically provided by the patient or their families Diabetes self-care activities in children and young adults were terribly compliant with taking medication however were solely moderately compliant to diet and self-glucose observance and least compliant to exercise. [9] Besides, individuals affected 
with the illness usually have inadequate information regarding the character of disease, its risk factors and associated complications. This lack of awareness is also the underlying issue touching attitudes and practices in the direction of its care. [10]

T1DM and its complications may disturb adolescents' living conditions over the years and may also affect their worth of being. Irresponsibility, difficulties in attaining autonomous control and a low degree of illness acceptance may hamper the everyday control of blood glucose levels. Overall, adolescents are more resilient to accommodating the disease than younger children since they no longer rely on their parents or caretakers for care and are accountable for their own wellbeing. [11]

Inadequate diabetic self-management remains a big drawback facing health care providers and populations in all settings. It has a bearing on the patient's morbidity, mortality plus increasing the costs of medication and laboratory tests, time consuming and effort of the care providers. [12] In disparity, patients who have sufficient self-management have healthier outcomes, live longer, appreciate a higher quality of life and feel pain of fewer symptoms \& negligible complications. [13]

School health nurse is in a strategic position in this specialized health care team because children spend long periods of time in their schools. So, she is the base stone in all aspect of diabetes management by assessing the students' needs and issues, giving suitable care, aiding diabetic adolescents and their families to deal with diabetes, keeping normal growth and development, attaining the most effective doable diabetes control and preventing complications. [14]

From analysis of the appropriate literature, it's evident that nationally there are several studies were conducted on diabetic children for a number of purposes including, studies in self-care practice of diabetic patients, [15] as well as here are generally restricted studies in El-Behaira Governorate. Accordingly, the aim of this study was to assess self-care practices of preparatory school students with Type 1 Diabetes Mellitus in EL Behira Governorate.

The research question of this study was:

What are the self-care practices of preparatory school students with type 1 Diabetes Mellitus in EL Behira governorate?

\section{Material and Methods}

\subsection{Research Design}

A descriptive research design was accompanied to achieve the aim of the study.

\subsection{Setting of Study}

Data were gained from governmental preparatory schools in El-Behira governorate.

\subsection{Subjects of Study}

The target population of this study was all students with type 1 diabetes mellitus registered in preparatory schools.

\subsection{Sample Size}

Sample size was 300 based on the prevalence of DM [16] $25 \%$ with a precision of $6 \%$, alpha error $=0.05$ and design effect $=2$, so sample size equal 300 students.

\subsection{Sampling Technique}

The schools' health insurance system in El-Behira governorate composed of three zones. Hence, subjects were chosen by multistage sampling technique through selecting a representative center from each zone based on the highest number of diabetic students found according to data collected from ElBehira health insurance system in the scholastic year 2015/2016, namely: - El Mhmodia (91 diabetic students), Damanhour (133 diabetic students) and El Rahmania (76 diabetic students), then all governmental preparatory schools belonged to above mentioned centers were enclosed in the study.

\subsection{Tool for Data Collection}

One tool was used to collect the data. A structured interview schedule was developed by the researchers after a thorough review of relevant literature and consists of three parts:-

\subsubsection{Part (1): Socio-demographic Data}

- Personal data:- age, sex, scholastic year, affiliated educational center, residence, birth order, siblings, attendance of training classes about diabetes mellitus and its management, type of housing and crowding index.

- Family data: - general characteristics of parents as age, occupation and level of education.

- Family Socioeconomic Status Scale (SES):- This scale was generated in 1983 by Fahmy and El-Sherbini. It was updated and validated by El-Gilany, El-Wehady and El-Wasify in 2012 in English language. [17] It was translated into Arabic language by the researchers. It contains seven domains as follows; education and culture for parents (30), occupation (10), family (10), family possessions (12), home sanitation (12), economic (5), health care (5). This creates a total SES score of 84 which was classified into four levels based on the quartiles:

$\begin{array}{ll}\text { Very low } & <25 \% \\ \text { Low } & 25 \%-<50 \% \\ \text { Middle } & 50 \%-<75 \% \\ \text { High } & \geq 75 \%\end{array}$

In case of death or retirement of husband or wife, the education and occupation before death or retirement were recorded.

\subsubsection{Part (2): Knowledge of Students Regarding Management of DM}

- Basic knowledge about diabetes: Definition, normal levels of fasting blood sugar, risk factors for diabetes and diabetes symptoms. 
- Knowledge about diabetes complications: Causes, symptoms and management of hyperglycaemia and hypoglycaemia.

- Knowledge about diabetes management: insulin, dietary pattern, physical exercise, foot care and Importance of follow up visits.

Scoring system for assessing knowledge of students entails 43 items and the correct answers were pre-determined according to the literature review. A score of (2) was given to the correct complete answer, a score of (1) for correct but incomplete answer and a score of (0) for the wrong or missed answers. So, the total knowledge score ranged from 0 - 86 and was categorized into: The total percentage of the total knowledge score was calculated as follows:-

- Poor knowledge $<43,(<50 \%)$

- Fair knowledge 43- <65, (50 - <75\%)

- Good knowledge $\geq 65$, $(\geq 75 \%)$.

\subsubsection{Part (3) Self-care Practices of Diabetes Mellitus:-}

- Insulin regimen: adherence for insulin use in controlling diabetes as regards to its type, dose, frequency and time of administration in relation to meals, patient's practice of self-insulin injection technique.

- management of complications: patient's practice about symptoms and measures of intervention in case of hyperglycemia or hypoglycemia, presence of diabetic patient identification card, previous hospitalization.

- Follow up activities: regularity of diabetic clinic visit, laboratory investigation\& its regularity.

- Dietary practices: practice about adherence to dietary regimen, meal planning method that the patient follows, number of meals per day, intake of snacks and regularity of meals times.

- Physical exercise: patient's adherence to physical exercise program and its regularity, precautions for practicing exercise as regards to its time of performance in relation to meals, blood glucose monitoring, insulin injection, changing sites of injection according to type of exercise performed and existence of diabetic coma afterward physical exercise.

- Personal hygiene and foot care: patient's hygienic practices as number of bathing per week, teeth care. foot care practices include, number of feet wash / day, daily feet observation, technique of feet dryness, wearing socks, type of socks, type of chose, wearing shoes at home.

Scoring system for assessing students' practice regarding diabetes includes 52 items. The items were scored as follows: A score "1" was given to performed practice, while, score "0" was given to incorrect or never done. The total practice score was obtained for each participant $(0$ 52). The percentage of total practice score was calculated as follows:

Poor practice $<26$ (less than $50 \%$ )

Fair practice $26-<39(50-<75 \%)$

Good practice $\geq 39(\geq 75 \%)$

\subsection{Methods}

- Basically, official letters from the faculty of nursing, university of Damanhour were directed to the responsible health authorities of study settings (Central Agency for Public Mobilization and Statistics CAPMAS, Directorate of Education and school health insurance in El-Behira governorate) to take their permission to conduct the study after explaining its purpose.

- Permission was obtained from Directorate of Education in El-Behira governorate to collect data from schools affiliated to the selected centers.

- Tool was developed by the researchers after a thorough review of relevant literature including data from students' health record.

- Tool was tested for reliability by test and retest and for validity by exposing them to a Jury composed of 3 experts in the field.

- The purpose and the nature of the study were clarified to students, for full cooperation. The researchers were able to interview about $8-10$ students daily. Each sheet took 15-20 minutes to be answered. Data was collected during the academic years $2016-2017$.

- A pilot study was conducted on a sample of randomly selected 15 students not included in the study to ascertain the clarity and applicability of the tool and to estimate the time needed to fill the study tools. Based on the result, the necessary modifications were made.

- The school administrator was met by the researchers to gain his cooperation after explaining the purpose of the study.

- Ethical consideration, Oral informed consent was obtained from every student included in the study after explanation of the purpose of the study.

- Each student was interviewed individually after establishing trustful relationship with him to gain his cooperation and confidence this was attained through explaining the purpose of the study, promising that the collected data will be used only for the purpose of the study and inform them about their unpaid sharing in the study.

\subsection{Statistical Analysis of the Data}

After collection of data, it was coded and transferred into special format, so as to be suitable for computer feeding. The Statistical Package for Social Sciences (SPSS) version 20 was utilized for data analysis and tabulation. Frequencies and percentage were calculated. The level of significance selected for this study was equal to 0.05 . The tests of significance used in this study were; chi-square test $\left(\mathrm{X}^{2}\right)$.

\section{Results}

Table 1 shows that $41.7 \%$ of the studied diabetic students aged between 12 to less than 13 years with a 
mean age $12.34 \pm 1.23$ and females represent $55.0 \%$ of them. The highest percent $(47.5 \%)$ of the studied diabetic students are enrolled in the third year of preparatory school and 40.3 of them were affiliated to Damanhour educational center. In addition, $56.0 \%$ of them were lived in rural areas and $68.3 \%$ of them are ranged as the first to the third child in their families with a mean of $2.65+$ 1.212 also $59.0 \%$ of them have one to three siblings with a mean of $2.42+1.32$. Finally, the majority $(84.3 \%)$ of the studied diabetic students is lived in owned housing and 90.3 of them haven't attended training classes about DM and its management.

Table 1. Distribution of the studied diabetic students according to their socio-demographic characteristics and training experience about DM

\begin{tabular}{|c|c|c|}
\hline Socio-demographic characteristics & $\begin{array}{c}\text { No. } \\
\mathbf{n}=\mathbf{3 0 0}\end{array}$ & $\%$ \\
\hline \multicolumn{3}{|l|}{ Age (in years) } \\
\hline $12-$ & 125 & 41.7 \\
\hline $13-$ & 95 & 31.6 \\
\hline$\geq 14$ & 80 & 26.7 \\
\hline Mean \pm SD & \multicolumn{2}{|c|}{$12.34 \pm 1.23$} \\
\hline \multicolumn{3}{|l|}{ Sex } \\
\hline Female & 165 & 55.0 \\
\hline Male & 135 & 45.0 \\
\hline \multicolumn{3}{|l|}{ Scholastic year } \\
\hline First & 113 & 26.6 \\
\hline Second & 70 & 25.9 \\
\hline Third & 117 & 47.5 \\
\hline \multicolumn{3}{|l|}{ Affiliated educational center } \\
\hline Damanhour & 121 & 40.3 \\
\hline El Mahmodia & 96 & 32.0 \\
\hline El Rahmania & 83 & 27.7 \\
\hline \multicolumn{3}{|l|}{ Residence } \\
\hline Rural & 168 & 56.0 \\
\hline Urban & 132 & 44.0 \\
\hline \multicolumn{3}{|l|}{ Birth order } \\
\hline $1-3$ & 205 & 68.3 \\
\hline $4-6$ & 95 & 31.7 \\
\hline Mean \pm SD & \multicolumn{2}{|c|}{$2.65 \pm 1.212$} \\
\hline \multicolumn{3}{|l|}{ Siblings } \\
\hline $1-3$ & 177 & 59.0 \\
\hline $4-6$ & 87 & 29.0 \\
\hline No siblings & 36 & 12.0 \\
\hline Mean \pm SD & \multicolumn{2}{|c|}{$2.42 \pm 1.32$} \\
\hline \multicolumn{3}{|l|}{ Type of housing } \\
\hline Owned & 253 & 84.3 \\
\hline Rented & 47 & 15.7 \\
\hline \multicolumn{3}{|l|}{$\begin{array}{l}\text { Attendance of training classes about DM and } \\
\text { its management }\end{array}$} \\
\hline Yes & 28 & 9.3 \\
\hline No & 272 & 90.7 \\
\hline
\end{tabular}

Table 2. portrays that 73.7 of the studied diabetic students' fathers aged between 30 to less than 50 years with a mean of $40.67 \pm 13.25$ and equal percent of them
(26.3\%) have either primary/read and write or secondary school education. In addition, $91.6 \%$ of them are working. Regarding the studied diabetic students' mothers, $63.4 \%$ of them aged between 40 to less than 60 years with a mean of $37.42 \pm 7.54$. Illiteracy was observed among $40.3 \%$ of them while $79.0 \%$ of them were housewives. The family crowding index is ranged between 1-3 among $95.3 \%$ of the studied diabetic students' families. Finally, the majority $(55.6 \%, 33.7 \%)$ of the studied diabetic students' families have either low or moderate socioeconomic status.

Table 2. Distribution of the studied diabetic student's parents according to their socio-demographic characteristics

\begin{tabular}{|c|c|c|}
\hline Socio-demographic characteristics & No. & $\%$ \\
\hline 1- Diabetic students' father* & $(n=285)$ & \\
\hline - Age (in years) & & \\
\hline$\bullet 30-$ & 210 & 73.7 \\
\hline • 50- & 55 & 19.3 \\
\hline$\bullet 70$ & 20 & 7.0 \\
\hline Mean + SD & \multicolumn{2}{|c|}{$40.67+13.25$} \\
\hline \multicolumn{3}{|l|}{ Education } \\
\hline - Illiterate & 60 & 21.0 \\
\hline - Primary/ Read \& write & 75 & 26.3 \\
\hline - Preparatory & 18 & 6.3 \\
\hline - Secondary & 75 & 26.3 \\
\hline • University & 57 & 20.0 \\
\hline \multicolumn{3}{|l|}{ Occupation } \\
\hline - Working & 261 & 91.6 \\
\hline - Not working & 24 & 8.4 \\
\hline 2-Diabetic students' mother\# & $(n=295)$ & \\
\hline \multicolumn{3}{|l|}{ Age (in years) } \\
\hline$\bullet 20-$ & 100 & 33.9 \\
\hline - 40- & 187 & 63.4 \\
\hline$\bullet 60$ & 8 & 2.7 \\
\hline Mean + SD & \multicolumn{2}{|c|}{$37.42+7.54$} \\
\hline \multicolumn{3}{|l|}{ Education } \\
\hline - Illiterate & 120 & 40.7 \\
\hline - Primary/ Read \& write & 36 & 12.2 \\
\hline - Preparatory & 15 & 5.1 \\
\hline - Secondary & 96 & 32.5 \\
\hline - University & 28 & 9.5 \\
\hline \multicolumn{3}{|l|}{ Occupation } \\
\hline - Housewife & 233 & 79.0 \\
\hline - working & 62 & 21.0 \\
\hline \multicolumn{3}{|l|}{ Crowding index } \\
\hline - $1-3$ & 286 & 95.3 \\
\hline - 4-6 & 14 & 4.7 \\
\hline Mean + SD & \multicolumn{2}{|c|}{$1.02+2.11$} \\
\hline \multicolumn{3}{|l|}{ Socioeconomic status (SES) } \\
\hline - Very low & 18 & 6.0 \\
\hline • Low & 167 & 55.6 \\
\hline - Moderate & 101 & 33.7 \\
\hline • High & 14 & 4.7 \\
\hline
\end{tabular}

$*$ Dead fathers $=15(5.0 \%)$ \# Dead mothers $=5(1.6 \%)$ 
Table 3. portrays that $33.0 \%$ of the studied diabetic students discovered the disease from four to less than six years with a mean of $3.52 \pm 0.983$. The main method of disease discovery is appearance classical manifestations of DM which was found among $81.7 \%$ of the studied diabetic students.

Table 3

\begin{tabular}{|l|c|c|}
\hline \multirow{2}{*}{ Diabetic history } & \multicolumn{2}{|c|}{ Total $\mathbf{n = 3 0 0}$} \\
\cline { 2 - 3 } & No & \% \\
\hline Disease discovery (in years) & & \\
$<2$ & 55 & 18.3 \\
$2-$ & 90 & 30.0 \\
$4-$ & 99 & 33.0 \\
$\geq 6$ & 56 & 18.7 \\
\cline { 2 - 3 } Mean + SD & \multicolumn{2}{|c|}{$3.52+0.983$} \\
\hline Method of disease discovery & \multicolumn{2}{|}{} \\
Classical manifestations & 245 & 81.7 \\
Coma & 40 & 13.3 \\
Check up & 15 & 5.0 \\
\hline
\end{tabular}

Figure 1. Displays that $76.7 \%$ of the studied diabetic students had poor total knowledge score and $22.3 \%$ of them had fair total knowledge score about different aspects of DM and its management.

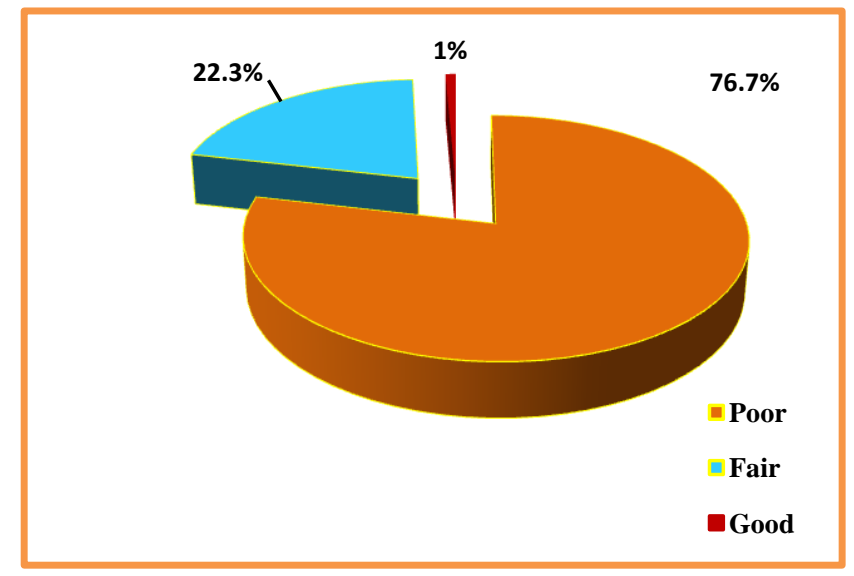

Figure 1. Distribution of the studied sample according to their total knowledge score about DM and its management.

Figure 2. Represents that $53.3 \%$ of the studied diabetic students have fair total self-care practices score whereas $29.7 \%$ and $17.0 \%$ of them have either poor or good total self-care practices respectively.

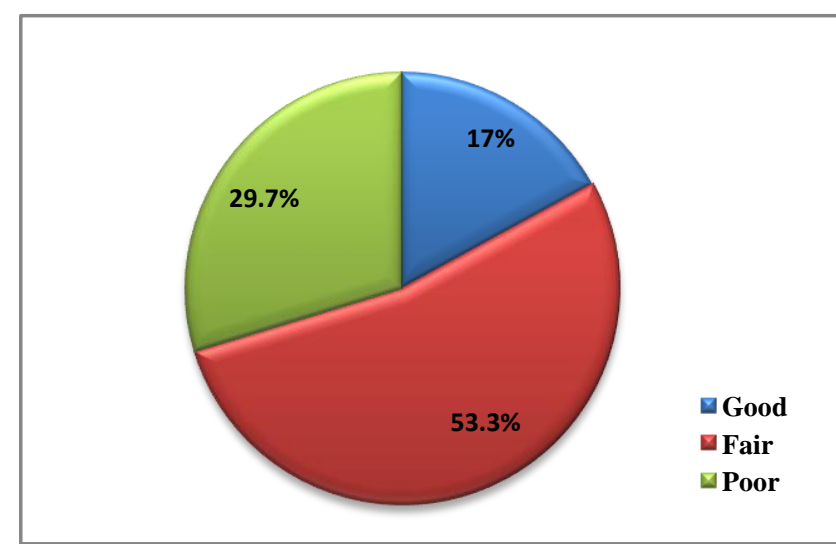

Figure 2. Distribution of the studied diabetic students according to their total self-care practice score

Table 4. Represents that most of the studied diabetic students have fair $(61.7 \%)$ or poor $(26.7 \%)$ insulin regimen practices. Self-monitoring of blood glucose level is mainly either fair $(55.3 \%)$ or poor $(38.0 \%)$ among the studied diabetic students. Fair practices during hyper or hypoglycemia and dietary regimen are observed among 55.0 and $79.7 \%$ of the studied diabetic students. In addition, most of them have fair $(58.7 \%)$ or poor $(37.3 \%)$ physical exercise practices. Most of the studied diabetic students have either fair $(57.0 \%)$ or good $(26.0 \%)$ follow up activities in addition; fair and poor personal hygiene and foot care practices are observed among $60.0 \%$ and $22.7 \%$ of them, respectively.

Table 5. Presents a statistically significant difference between the studied diabetic students' total knowledge score and their age $(\mathrm{P}=0.008)$, previous hospitalization due to hypo/hyperglycemic coma $(\mathrm{P}=0.002)$ and duration of disease discovery $(\mathrm{P}=0.050)$ and socioeconomic status ( $\mathrm{P}=0.037)$. On the other hand, no statistically significant difference is found between the studied diabetic students' total knowledge score and their gender $(\mathrm{P}=0.254)$, residence $(\mathrm{P}=0.846)$, birth order $(\mathrm{P}=0.411)$ and method of disease discovery $(\mathrm{P}=0.844)$.

Table 6. Reveals a statistically significant difference between the studied diabetic students' total self-care practice score and their gender $(\mathrm{P}=0.006)$, age $(\mathrm{P}=0.004)$, previous hospitalization due to hypo/hyperglycemic coma $(\mathrm{P}=0.032)$ and duration of disease discovery $(\mathrm{P}=0.047)$. On the other hand, no statistically significant difference is found between the studied diabetic students' total self-care practice score and their residence $(\mathrm{P}=0.132)$, birth order $(\mathrm{P}=0.236)$ and method of disease discovery $(\mathrm{P}=0.467)$.

Table 4. Self-care practices' scores of the studied diabetic students

\begin{tabular}{|c|c|c|c|c|c|c|}
\hline \multirow{2}{*}{ Practices } & \multicolumn{2}{|c|}{ Good } & \multicolumn{2}{|c|}{ Fair } & \multicolumn{2}{|c|}{ Poor } \\
\hline & No & $\%$ & No & $\%$ & No & $\%$ \\
\hline Insulin regimen & 35 & 11.7 & 185 & 61.7 & 80 & 26.7 \\
\hline Self-monitoring of blood glucose level (SMBG) & 20 & 6.7 & 166 & 55.3 & 114 & 38.0 \\
\hline Managing practices during hyper/ hypoglycemia & 46 & 15.3 & 165 & 55.0 & 89 & 29.7 \\
\hline Dietary regimen & 15 & 5.0 & 239 & 79.7 & 46 & 15.3 \\
\hline Physical exercise & 12 & 4.0 & 176 & 58.7 & 112 & 37.3 \\
\hline Follow up & 78 & 26.0 & 171 & 57.0 & 51 & 17.0 \\
\hline Personal hygiene and foot care & 52 & 17.3 & 180 & 60.0 & 68 & 22.7 \\
\hline
\end{tabular}


Table 5. Relationship between the studied diabetic students' total knowledge score and certain students' characteristics

\begin{tabular}{|c|c|c|c|c|c|c|c|c|c|c|}
\hline \multirow[b]{2}{*}{ Students' characteristics } & \multirow{2}{*}{ Knowledge score } & \multicolumn{2}{|c|}{ Good } & \multicolumn{2}{|c|}{ Fair } & \multicolumn{2}{|c|}{ Poor } & \multicolumn{2}{|c|}{ Total } & $X^{2}$ (P value) \\
\hline & & No & $\%$ & No & $\%$ & No. & $\%$ & No & $\%$ & \multirow{3}{*}{$(0.254)$} \\
\hline \multirow{2}{*}{ Gender } & Male & 0 & 0.0 & 25 & 18.5 & 110 & 81.5 & 135 & 45.0 & \\
\hline & Female & 2 & 1.2 & 36 & 21.8 & 127 & 77.0 & 165 & 55.0 & \\
\hline \multirow{3}{*}{ Age } & $12-$ & 0 & 0.0 & 24 & 18.2 & 108 & 81.8 & 132 & 44.0 & \multirow{3}{*}{$\left(0.008^{*}\right)$} \\
\hline & $13-$ & 2 & 2.3 & 19 & 22.1 & 65 & 75.6 & 86 & 28.7 & \\
\hline & $\geq 14$ & 0 & 0.0 & 19 & 23.2 & 63 & 76.8 & 82 & 27.3 & \\
\hline \multirow{2}{*}{ Residence } & Rural & 2 & 0.6 & 38 & 21.4 & 128 & 78.0 & 168 & 56.0 & \multirow{2}{*}{$(0.846)$} \\
\hline & Urban & 1 & 0.7 & 24 & 18.2 & 107 & 81.1 & 132 & 44.0 & \\
\hline \multirow{2}{*}{ Birth order } & $1-3$ & 1 & 0.5 & 40 & 19.5 & 164 & 80.0 & 205 & 68.3 & \multirow{2}{*}{$(0.411)$} \\
\hline & $4-6$ & 2 & 2.1 & 24 & 25.3 & 69 & 72.6 & 95 & 31.7 & \\
\hline \multirow{4}{*}{$\begin{array}{l}\text { Hospitalization due to } \\
\text { hypo/hyperglycemic coma }\end{array}$} & $1-$ & 1 & 0.9 & 26 & 22.0 & 91 & 77.1 & 118 & 39.4 & \multirow{4}{*}{$(0.002 *)$} \\
\hline & 4- & 1 & 6.2 & 2 & 12.5 & 13 & 81.3 & 16 & 5.3 & \\
\hline & $\geq 7$ & 0 & 0.0 & 5 & 71.4 & 2 & 28.6 & 7 & 2.3 & \\
\hline & No & 0 & 0.0 & 29 & 18.2 & 130 & 81.8 & 159 & 53.0 & \\
\hline \multirow{4}{*}{ Duration of disease discovery } & $<2$ & 6 & 10.9 & 15 & 27.3 & 34 & 61.8 & 55 & 18.3 & \multirow{4}{*}{$(0.050 *)$} \\
\hline & $2-$ & 10 & 11.1 & 20 & 22.2 & 60 & 66.7 & 90 & 30.0 & \\
\hline & 4- & 70 & 70.7 & 28 & 28.3 & 1 & 1.0 & 99 & 33.0 & \\
\hline & $\geq 6$ & 40 & 71.4 & 15 & 26.8 & 1 & 1.8 & 56 & 18.7 & \\
\hline \multirow{3}{*}{ Method of disease discovery } & Clinical manifestations & 2 & 0.8 & 54 & 22.1 & 189 & 77.1 & 245 & 81.7 & \multirow{3}{*}{$(0.844)$} \\
\hline & Occurrence of coma & 2 & 5.0 & 8 & 20.0 & 30 & 75.0 & 40 & 13.3 & \\
\hline & Check up & 8 & 53.4 & 5 & 33.3 & 2 & 13.3 & 15 & 5.0 & \\
\hline \multirow{4}{*}{ Socio-economic status } & Very low & 4 & 22.2 & 6 & 33.3 & 8 & 44.5 & 18 & 6.0 & \multirow{4}{*}{$(0.037 *)$} \\
\hline & Low & 30 & 18.0 & 79 & 47.3 & 58 & 34.7 & 167 & 55.6 & \\
\hline & Moderate & 28 & 27.7 & 51 & 50.5 & 22 & 21.8 & 101 & 33.7 & \\
\hline & High & 7 & 50.0 & 5 & 35.7 & 2 & 14.3 & 14 & 4.7 & \\
\hline
\end{tabular}

Table 6. Relationship between the studied diabetic students' total self-care practice and certain students' characteristics

\begin{tabular}{|c|c|c|c|c|c|c|c|c|c|c|}
\hline \multirow[b]{2}{*}{ Students' characteristics } & \multirow[t]{2}{*}{ Total self-care practice score } & \multicolumn{2}{|c|}{ Good } & \multicolumn{2}{|c|}{ Fair } & \multicolumn{2}{|c|}{ Poor } & \multicolumn{2}{|c|}{ Total } & \multirow{3}{*}{$\begin{array}{c}\mathbf{X}^{2} \text { (P value) } \\
\left(0.006^{*}\right)\end{array}$} \\
\hline & & No & $\%$ & No & $\%$ & No. & $\%$ & No. & $\%$ & \\
\hline \multirow{2}{*}{ Gender } & Male & 44 & 32.6 & 68 & 50.4 & 23 & 17.0 & 135 & 45.0 & \\
\hline & Female & 32 & 19.3 & 97 & 58.9 & 36 & 21.8 & 165 & 55.0 & \\
\hline \multirow{3}{*}{ Age } & $12-$ & 24 & 18.2 & 64 & 48.5 & 44 & 33.3 & 132 & 44.0 & \multirow{3}{*}{$(0.004 *)$} \\
\hline & $13-$ & 0 & 0 & 19 & 22.1 & 67 & 77.9 & 86 & 28.7 & \\
\hline & $\geq 14$ & 2 & 2.4 & 20 & 62.5 & 60 & 73.2 & 82 & 27.3 & \\
\hline \multirow{2}{*}{ Residence } & Rural & 44 & 26.2 & 80 & 47.6 & 44 & 26.2 & 168 & 56.0 & \multirow{2}{*}{$(0.132)$} \\
\hline & Urban & 30 & 22.7 & 82 & 62.1 & 20 & 15.2 & 132 & 44.0 & \\
\hline \multirow{2}{*}{ Birth order } & $1-3$ & 9 & 4.4 & 36 & 17.6 & 160 & 78.0 & 205 & 68.3 & \multirow{2}{*}{$(0.236)$} \\
\hline & $4-6$ & 7 & 7.4 & 20 & 21.0 & 68 & 71.6 & 95 & 31.7 & \\
\hline \multirow{4}{*}{$\begin{array}{l}\text { Hospitalization due to } \\
\text { hypo/hyperglycemic coma }\end{array}$} & $1-$ & 25 & 0.9 & 69 & 22.0 & 24 & 77.1 & 118 & 39.4 & \multirow{4}{*}{$(0.032 *)$} \\
\hline & $4-$ & 2 & 6.2 & 8 & 12.5 & 6 & 81.3 & 16 & 5.3 & \\
\hline & $\geq 7$ & 0 & 0.0 & 6 & 71.4 & 1 & 28.6 & 7 & 2.3 & \\
\hline & No & 37 & 0.0 & 81 & 18.2 & 41 & 81.8 & 159 & 53.0 & \\
\hline \multirow{4}{*}{ Duration of disease discovery } & $<2$ & 2 & 3.6 & 40 & 72.8 & 13 & 23.6 & 55 & 18.3 & \multirow{4}{*}{$(0.047 *)$} \\
\hline & $2-$ & 10 & 11.1 & 58 & 64.4 & 22 & 24.5 & 90 & 30.0 & \\
\hline & $4-$ & 10 & 10.1 & 66 & 66.7 & 23 & 23.2 & 99 & 33.0 & \\
\hline & $\geq 6$ & 7 & 12.5 & 39 & 69.6 & 10 & 17.9 & 56 & 18.7 & \\
\hline \multirow{3}{*}{ Method of disease discovery } & Clinical manifestations & 53 & 21.6 & 132 & 53.9 & 60 & 24.5 & 245 & 81.7 & \multirow{3}{*}{$(0.467)$} \\
\hline & Occurrence of coma & 4 & 10.0 & 26 & 65.0 & 10 & 25.0 & 40 & 13.3 & \\
\hline & Check up & 3 & 20.0 & 10 & 66.7 & 2 & 13.3 & 15 & 5.0 & \\
\hline
\end{tabular}


Table 7. Relationship between the studied diabetic students' total self-care practices score and their family characteristics

\begin{tabular}{|c|c|c|c|c|c|c|c|c|c|c|}
\hline \multirow[b]{2}{*}{ Family characteristics } & \multirow[t]{2}{*}{ Total self-care practice score } & \multicolumn{2}{|c|}{ Good } & \multicolumn{2}{|c|}{ Fair } & \multicolumn{2}{|c|}{ Poor } & \multicolumn{2}{|c|}{ Total } & \multirow[t]{2}{*}{$\mathbf{X}^{2}$ (P value) } \\
\hline & & No. & $\%$ & No. & $\%$ & No. & $\%$ & No. & $\%$ & \\
\hline \multirow{5}{*}{ Father's education } & Illiterate & 8 & 13.3 & 30 & 50.0 & 22 & 36.7 & 60 & 21.0 & \multirow{5}{*}{$\left(0.022^{*}\right)$} \\
\hline & Primary/read and write & 14 & 18.7 & 45 & 60.0 & 16 & 8.0 & 75 & 26.3 & \\
\hline & Preparatory & 6 & 33.3 & 11 & 61.1 & 1 & 5.6 & 18 & 6.3 & \\
\hline & Secondary & 11 & 14.7 & 34 & 45.3 & 30 & 40.0 & 75 & 26.3 & \\
\hline & University & 11 & 19.3 & 31 & 54.4 & 15 & 26.3 & 57 & 20.0 & \\
\hline \multirow{5}{*}{ Mother's education } & Illiterate & 35 & 29.2 & 65 & 54.2 & 20 & 16.7 & 120 & 40.7 & \multirow{5}{*}{$\left(0.006^{*}\right)$} \\
\hline & Primary/read and write & 4 & 11.1 & 25 & 69.5 & 7 & 19.4 & 36 & 12.2 & \\
\hline & Preparatory & 2 & 13.3 & 9 & 60.0 & 4 & 26.7 & 15 & 5.1 & \\
\hline & Secondary & 14 & 14.6 & 51 & 53.1 & 31 & 32.3 & 96 & 32.5 & \\
\hline & University & 6 & 21.4 & 13 & 46.5 & 9 & 32.1 & 28 & 9.5 & \\
\hline \multirow{2}{*}{ Mother's working condition } & Working & 12 & 19.3 & 37 & 59.7 & 13 & 21.0 & 62 & 21.0 & \multirow{2}{*}{$(0.034 *)$} \\
\hline & House wife & 18 & 7.7 & 160 & 68.7 & 55 & 23.6 & 233 & 79.0 & \\
\hline \multirow{2}{*}{ Crowding index } & $1-3$ & 32 & 11.2 & 192 & 67.1 & 62 & 21.7 & 286 & 95.3 & \multirow{2}{*}{0.746} \\
\hline & $4-6$ & 3 & 21.4 & 6 & 42.9 & 5 & 35.7 & 14 & 4.7 & \\
\hline \multirow{4}{*}{ Socio-economic status } & Very low & 3 & 16.7 & 6 & 33.3 & 9 & 50.0 & 18 & 6.0 & \multirow{4}{*}{$(0.003 *)$} \\
\hline & Low & 27 & 16.2 & 80 & 47.9 & 60 & 35.9 & 167 & 55.6 & \\
\hline & Moderate & 30 & 29.7 & 50 & 49.5 & 21 & 20.8 & 101 & 33.7 & \\
\hline & High & 6 & 42.9 & 5 & 35.7 & 3 & 21.4 & 14 & 4.7 & \\
\hline
\end{tabular}

Table 8. Relationship between the studied diabetic students' total self-care practices and total knowledge scores

\begin{tabular}{|c|c|c|c|c|c|c|c|c|c|c|}
\hline \multirow{2}{*}{ Knowledge } & \multirow[t]{2}{*}{ Self- care } & \multicolumn{2}{|c|}{ Good } & \multicolumn{2}{|c|}{ Fair } & \multicolumn{2}{|c|}{ Poor } & \multicolumn{2}{|c|}{$\begin{array}{c}\text { Total } \\
\mathbf{N}=\mathbf{3 0 0} \\
\end{array}$} & $X^{2}$ (P value) \\
\hline & & No. & $\%$ & No. & $\%$ & No. & $\%$ & No. & $\%$ & \multirow{4}{*}{$\left(0.045^{*}\right)$} \\
\hline Good & & 0 & 0.0 & 2 & 100.0 & 0 & 0.0 & 2 & 0.7 & \\
\hline Fair & & 9 & 14.5 & 37 & 59.7 & 16 & 25.8 & 62 & 20.7 & \\
\hline Poor & & 55 & 23.3 & 125 & 53.0 & 56 & 23.7 & 236 & 78.6 & \\
\hline
\end{tabular}

$\mathrm{X}^{2}$ : chi-square test, *Significant at 0.05 level.

Table 7. Portrays a statistically significant difference between the studied diabetic students' total self-care practice score and their father's education $(\mathrm{P}=0.022)$, mothers' education $(\mathrm{P}=0.006)$ and mother's working condition $(\mathrm{P}=0.034)$ and socioeconomic status $(\mathrm{P}=0.003)$ however no statistically significant difference was found in relation to their crowding index $(\mathrm{P}=0.746)$.

Table 8. Illustrates a statistically significant difference between the studied diabetic students' total self-care practice and total knowledge score a $(\mathrm{X} 2=0.045)$.

\section{Discussion}

Self-management is crucial for better physical and psychological consequences of the diabetes especially among the growing children. They should be supported to promote their autonomy and self-care capacity. Hence, need-based self-care education is essential for better glycemic control and prevention or even reduces both the onset and progression of diabetes complications. [18,19]

The present study depicted that most of the studied diabetic students had fair $(53.3 \%)$ and poor $(28.7 \%)$ total self-care practice whereas $17.0 \%$ of them had good selfcare practices. This can be ought to the young age of the studied sample where $41.7 \%$ of them aged between $12<13$ years where this early adolescence period is an open stage for outside world and negative peer influence on their health behaviors. Furthermore, there is prevalent poor knowledge level (76.7\%) among the studied diabetic students about DM and its management. This was coupled with the low socioeconomic status apparent among 55.6\% of them.

Concurred findings were shown by four recent studies. First, Lin K et al (2016) [20] found that most of the studied Chinese diabetic children had poor self-care behaviors. Second, Madian A and Ismail S (2015) [21] found that all the studied Egyptian diabetic students had either fair or poor total self-care practice score. Third, Hackworth N et al (2013) [22] revealed suboptimal diabetes self-care regimes among their studied Australian diabetic adolescents. Fourth, Vasli et al (2009) [23] asserted that three quarters of the studied Iranian diabetic children had average to weak self-care practices score.

Contradictory results were shown by Salehi $\mathrm{S}$ et al (2014) [24] explored that their studied Iranian diabetic children had both good and very good self-care practices scores. This could be justified by the wider age range of the studied diabetic students (11-17 years) in this contradictory study than the current one (12-15 years) 
which give chance for gaining knowledge and experience in managing the disease.

Insulin therapy is the mainstay of TIDM management. The current study portrayed that most of the studied diabetic students had either fair $(61.7 \%)$ or poor $(26.7 \%)$ insulin regimen practices. This explained via the irregular insulin use by many of the studied diabetic students due to forgetfulness or irregularity of meal timing. In addition, more than two fifths of them didn't practice self-insulin injection due to fear of injection or didn't know how to practice self-insulin injection. Moreover, they didn't change the insulin injection sites as they accustomed to certain body parts. This highlighted the urgent need to teach the diabetic children the correct self-insulin injection technique. Similarly, Salehi S et al (2014) [23] revealed that nearly one quarter of the studied Iranian diabetic children had average to weak insulin regimen practices despite that most of them had good insulin injection technique. They clarified that the studied diabetic children neither considered circular insulin injection to different parts of body nor adjust insulin dose in relation to physical activity and diet.

Contrast findings were revealed by two studies. First, Attia A and Hassan E (2017) [24] reported that none of their studied Egyptian diabetic children had adequate insulin injection practices. This could be justified by the young age of their studied sample (6-14 years). Second, Greene $M$ et al (2010) [25] asserted that their studied American diabetic adolescents had high insulin regimen practices where they adjusted insulin in relation to meal. This can be ascribed to the used approach in this contradictory study of self-rating of their self-care behaviors which can be less objective.

Self-Monitoring of Blood Glucose (SMBG) is a critical aspect of TIDM management and increasing its frequency is greatly linked to improved clinical outcomes. [27] The current study portrayed that most of the studied diabetic students had either fair $(55.3 \%)$ or poor $(38.0 \%)$ practice of SMBG where $40.0 \%$ of them fear from needle stick injury and neither do not have the Gluco-Check apparatus nor know how to use it. Concurred findings were portrayed by two recent evidences. First, Tanjia et al (2016) [28] revealed that nearly two thirds of their Asian diabetic participants never performed SMBG owing to the appeared lack of knowledge and feeling of unnecessary of doing it. Second, Santiprabhob J et al (2012) [29] they also portrayed poor practices of SMBG among most of their Asian diabetic adolescent.

Recent evidence showed that children with early onset of diabetes are at highest risk for disturbance of their cognitive function and neuropsychological skills, but the corresponding role of either hypo or hyperglycemia are still under investigation. Thus, the management practices for hypo/hyperglycemia must be scrutinized where both the diabetic children and their caregivers must be aware of their symptomatic management practices. [30] The present study explored that most of the studied diabetic students had either fair $(55.0 \%)$ or poor $(29.7 \%)$ practice during hyper or hypoglycemia. This was evidenced by that $40.0 \%$ of them had previous hospitalization due to hypo/hyperglycemic coma. Most of them kept neither a sugar source nor identification card. In addition, they increase/decrease insulin dose by themselves in response to high/low blood glucose level whereas less than one fifth of them take no action to manage their hypo/hyperglycemic attack.

Consistent findings were revealed by Santiprabhob J et al (2012), [29] as they portrayed fair managing practices for hypo/hyperglycemic attacks among more than half of their Asian diabetic children who treated themselves with sweetened drink and carried sugar source when they traveled.

In contrast, Salehi $\mathrm{S}$ et al (2014) [24] showed that nearly two fifths of their studied Iranian diabetic children had good management activities for hypo/ hyperglycemia despite that half of them had no identification card which is congruent with the current study. This can be explained through the lack of knowledge of their participants about the importance of such card. The discrepancy between the current and this contrasting study could be ascribed to the smaller sample size (99) and the wider age rang (10-18 years) than the current study.

Dietary regimen is the backbone for proper metabolic control of T1DM along with insulin therapy and physical exercise. [31] The current study represented that $79.7 \%$ and $15.3 \%$ of the studied diabetic students had either fair or poor dietary practices, respectively. Evidently, they were found to have certain misconceptions about diabetic diet as reducing complex carbohydrates (35.1\%) and unimportance of diabetic meal plan $(34.6 \%)$. In addition, $21.7 \%$ of them did not adherent to diabetic diet regimen and took no snacks $(30.3 \%)$. Similar findings were revealed by a novel study of Silva A et al (2016), [32] as they reported that dietary compliance was the main encountered problem among their studied Brazilian diabetic adolescents.

Physical exercise is enormously imperative for diabetes management owing to its crucial role in reducing blood glucose level. [33] The present study illustrated that most of the studied diabetic students had either fair $(58.7 \%)$ or poor $(37.3 \%)$ physical exercise practices where more than half of them didn't participate in any sport. In addition, similar percent $(69.2 \%)$ of them didn't eat or check blood glucose level in relation to any physical activity and $92.3 \%$ of them didn't change insulin injection site based on type of physical activity.

Similar findings were reported by two recent studies. First, Attia A and Hassan E (2017) [25] reported that more than one fifth of the studied Egyptian diabetic children had low physical exercise practices where they neither participated in any exercise program nor controlled their blood glucose before any physical activity. Second, Santiprabhob J et al (2012) [29] showed that most of their respondents had poor physical exercise practices as they rarely walked or did household tasks for at least 30 minutes, checked their blood glucose prior to exercising and ate extra snacks when exercising vigorously. In contrast, Silva A et al (2016) [32] showed that their studied Brazilian diabetic students had adequate physical exercise practices were more than three quarters of them participate in one or more sports. This could be ascribed to emphasizing of the author of this contradictory study on exercise performance and not taking into consideration blood glucose level monitoring and controlling practices in relation to exercise performance.

Follow up activities can be considered as a monitoring 
scale for diabetes management practices. [21] The current study represented that $57.0 \%$ and $26.0 \%$ of the studied diabetic students had either fair or good follow up activities, respectively; where most of them reported regular visiting of the diabetic clinic and performing of laboratory investigation especially fasting and post prandial blood glucose level. This could be explained by their monthly need for insulin therapy which is basically determined based on their blood glucose level through the students' health insurance clinic.

Corresponding evidence was illustrated by two recent studies. First, Tanjia et al (2016) [28] claimed that most of their studied Asian diabetic children reported that they always attend DM clinic or visit their doctor for follow up and performed urine examination. Second Madian A and Ismail S (2015) [21] illustrated that most of their Egyptian diabetic children had regular follow up activities.

Proper personal hygiene especially foot care is essential aspect for diabetes management as high glycemic level could increase the risk of infection. [34] The current study confirmed that most of the studied diabetic students had either fair $(60.0 \%)$ or poor $(22.7 \%)$ general personal hygiene and foot care practices, respectively where more than two fifths of them didn't observe their feet regularly with improper dryness technique and had inadequate number of bathing and teeth brushing.

Conformable results were asserted by a study done by, Desalu O et al (2011), [35] as they reported that majority of their studied Nigerian diabetic patients had either satisfactory or poor foot care practice and only $10.2 \%$ of them had good practice of foot care in relation to regularity of feet inspection, washing and dryness and inspection of inside of their footwear.

Several demographic and socio-economic factors can contribute positively to facilitate self-care performance in diabetic children. Hence, it can be mediated through certain individual or familial factors. [36] Regarding individual factors, the present study portrayed a significant difference between the studied diabetic students' total self-care practice score and their gender, age, previous hospitalization due to hypo or hyperglycemic coma and duration of disease discovery. Identical findings were portrayed by two novel studies. First, Gomes $M$ et al (2018) [37] revealed that self-management of T1DM among young people was associated with higher age, more years of school attendance, lower reported rates of hypoglycemia and better glycemic control. Second, Ryan M (2014) [38] found that age was significantly correlated with diabetes self-care practices score. Lin k et al (2016) [20] revealed no significant difference between students' age and their self-care behaviors. The author justified that by the small numbers of the studied diabetic children in their cohort study.

Regarding family factors, the current study depicted a significant difference between the studied diabetic students' total self-care practice score and their father's education, mothers' education, mother's working condition and socio-economic status. Consistent findings were illustrated by Soheilipour F et al (2015), [39] as they suggested that higher mothers' awareness is positively correlated with better blood sugar control in their children which was mainly mediated by their socioeconomic status. Furthermore, Niba LL et al (2017) [40] asserted that minimal maternal involvement as a primary caregiver in self-care practices of their diabetic children were significantly associated with poor outcome.

Evidence portrayed that knowledge about diabetes and its management can mediate the relationship between self-care practices and glycemic control. [33] The present study revealed a statistically significant difference between the studied diabetic students' total self-care practice and total knowledge score. Correspondingly, Basu $S$ et al (2017) [41] portrayed that low knowledge level about DM was a predictor of poor self-care practices and associated glycemic control. Converse findings were shown by Onuoha PH (2017) [42] who revealed that the total knowledge score was un proportionate to the level of self-care among their participants where the study showed that most of them had moderate to high total knowledge score with inadequate level of self-care practices.

Knowledge can be considered as a basic component for behavior change as it plays an essential role in shaping individuals' participation in proper health maintenance practices along with health-related beliefs. [43] The present study displayed that almost all the studied diabetic students had either poor $(76.7 \%)$ or fair $(22.3 \%)$ total knowledge score about DM and its management aspects. This could be attributed to the young age of the studied diabetic students (12-15 years) and the lower duration of disease discovery were about one third of the studied diabetic students discovered the disease from $4<6$ years with a mean of $3.52 \pm 0.983$. Specifically, those years were mainly during primary education were children were mainly dependent on their mothers in all aspects of their life including diabetes management.

Similarly, Basu S et al (2017) [41] and Tanjia et al (2016) [28] portrayed low knowledge level among their participants in relation to diabetes and almost all aspects of its management. Conversely, Flora $\mathrm{M}$ and Gameiro $\mathrm{M}$ (2016) [44] found that most of their studied diabetic adolescents had good overall knowledge level about diabetes and its management however less than one fifth of them had low total knowledge score especially in relation to insulin regimen and management of disease's complications. Such discrepancy in the knowledge level between the current and these contradictory study could be attributed to previous attendance of the studied diabetic adolescents of DM educational programs which is incommensurable to the current study were majority of the participants didn't attend diabetes educational classes.

Moreover, the current study revealed a significant difference between the studied diabetic students' total knowledge score and their previous hospitalization due to hypo/hyperglycemic coma, duration of disease discovery and socioeconomic status. Concurred findings were shown by four recent studies. First, Gomes M et al (2018) [37] portrayed that DM related knowledge was associated with lower rates of severe hypoglycemia, more years of school attendance and economic status. Second, Moskovitz A et al (2018) [45] revealed that better knowledge score of the studied diabetic adolescents was significantly associated with longer duration of contracting the disease. Third, Flora M and Gameiro M (2016) [44] found positive correlation between the studied diabetic adolescents' total knowledge level and their age. Fourth, Desalu O et al 
(2011) [35] showed that illiteracy and low socioeconomic status were significantly associated with poor knowledge level of diabetes self-care.

\section{Conclusion}

The results of the present study concluded that the highest percentage of the studied diabetic preparatory school students have either fair or poor self-care practices in all aspects of DM management; insulin regimen practices, SMBG, managing practices during hyper/hypoglycemia, dietary regimen, physical exercise, personal hygiene and foot care practices except follow up practices which were found to be fair or good among most of them. A significant difference was revealed between the studied diabetic students' total score of knowledge and self-care practices. In addition, diabetic students' age, previous hospitalization due to hypo/hyperglycemic coma, duration of disease discovery and socioeconomic status were significantly varied among them in relation to their total knowledge and self-care practices scores. Moreover, diabetic students' gender, father's education, mothers' education, mother's working condition were significantly varied among them in relation to their total self-care practices score.

\section{Recommendations}

- Developing hotlines, mass media campaigns for dissemination of accurate information about juvenile diabetes; classical manifestations, complications and self-care management

- Establish community based support groups for diabetic children and adolescents

- Establishing an accurate surveillance and documentation system of diabetic students for easy accessibility and provision of accurate information to improve their self-care capacity.

- Provide in-service training programs for school health nurses about diabetes management and emergency measures in school for either hypo or hyperglycemia.

- Motivate diabetic students' parents to participate actively in management of their diabetic children by encouraging them to attend parents' council in schools regularly.

\subsection{Further Research}

- Assessment of diabetic children's perceived barriers to self-care behaviors

- Impact of diabetes on scholastic achievement of the diabetic students.

- Quality of life of diabetic children's parents.

\section{References}

[1] El-Ziny M, Salem NA, El-Hawary AK, Chalaby NM and Elsharkawy AA. Epidemiology of childhood type 1 diabetes mellitus in Nile Delta, northern Egypt-a retrospective study.
Journal of Clinical Research in Pediatric Endocrinology. 2014; 6(1): 9-15.

[2] International Diabetes Federation. The IDF Diabetes Atlas. 7th ed. http://www.idf.org/idf-diabetes-atlas-seventh-edition. Date of last access 23th April 2018. Brussels: International Diabetes Federation; 2015.

[3] International Diabetes Federation. The IDF Diabetes Atlas. 5th ed. http://www.idf.org/idf-diabetes-atlas-fifth-edition. Date of last access 23th April 2018. Brussels: International Diabetes Federation; 2011.

[4] International Diabetes Federation. Last accessed 2/6/2018 Available at:

https://www.idf.org/our-network/regions-members/middle-eastand-north-africa/members/34-egypt.html.

[5] Dhatariya, K.K., Nunney, I., Higgins, K., Sampson, M.J., and Iceton, G. A national survey of the management of diabetic ketoacidosis in the UK in 2014. Diabetic Med. 2016; 33: 252-260.

[6] kim W, Hong JP, Ho Yoo J. Emotional and Behavioral Problems and Glycemic Control in Adolescents with Type 1 and Type 2 Diabetes. Journal of Psychiatry. 2017; 18(2): 1-5.

[7] Alrahbi H. Diabetes self-management (DSM) in Omani with type2 diabetes. International Journal of Nursing Sciences. 2014; 1(4): 352-9.

[8] Al Bimani ZS, Khan SA, David P. Evaluation of T2DM related knowledge and practices of Omani patients. Saudi Pharmaceutical Journal. 2015; 23(1): 22-7.

[9] Karimi, F., etal. Self-care behavior of type 2 diabetes mellitus patients in Bandar Abbas in 2015. Electron Physician. 2017 Nov; 9(11): 5863-5867

[10] Costa LM, and Vieira SE. Quality of life of adolescents with type 1 diabetes. Clinics (Sao Paulo). 2015; 70(3): 173-179.

[11] Dhatariya, K.K. and Umpierrez, G.E. Guidelines for management of diabetic ketoacidosis: time to revise? Lancet Diabetes Endocrinol. 2017; 5: 321-323.

[12] Wang, Y., Desai, M., Ryan, P.B., DeFalco, F.J., Schuemie, M.J., Stang, P.E. et al. Incidence of diabetic ketoacidosis among patients with type 2 diabetes mellitus treated with SGLT2 inhibitors and other antihyperglycemic agents. Diabetes Res Clin Pract. 2017; 128: 83-90.

[13] Jacobsen, L. Understanding pre Type 1 Diabetes: The Key to Prevention. Front Endocrinal (Lausanne). 2018; 9: 70

[14] Hilal H. Alrahbi, Said A. Alghenaimi. Factors influencing diabetes self-management among Omani patients with type-2 diabetes: Patients' perspectives. Journal of Nursing Education and Practice .2017; 7(12).

[15] Abdela NH. Quality of Life, Self-care Activities, and Diabetes control among school aged children. International Journal of Research-Granthaalayah 2017; 5(1): 1-11.

[16] The Central Agency for Public Mobilization and Statistics (CAPMAS), Elbehira Governorate, 2015.

[17] El-Gilany A, El-Wehady A, El-Wasify M. Updating and validation of the socioeconomic status scale for health research in Egypt. Eastern Mediterranean Health Journal 2012; 18(9): 962-68.

[18] Pelicand J. Fournier C. Le Rhun A. Aujoulat I. Self-care support in pediatric patients with type 1diabetes: bridging the gap between patient education and health promotion? A review. Blackwell Publishing Ltd; Health Expectations 2013 (18): 303-11.

[19] Wherrett D, Ho J, Huot C, Legault L, Nakhla M, Rosolowsky E.Type 1 Diabetes in Children and Adolescents. Diabetes Canada Clinical Practice Guidelines Expert Committee. Can J Diabetes 2018; (42): 234-46.

[20] Lin K, Yang X, Yin G, Lin S. Diabetes Self-Care Activities and Health-Related Quality-of-Life of individuals with Type 1 Diabetes Mellitus in Shantou, China. Journal of International Medical Research 2016; 44(1) 147-56.

[21] Madian A and Ismail S. Self-Care Knowledge and Practices among Diabetic School Students in El- Behaira Governorate. 2014 http://www.damanhour.edu.eg/pdf/186/FINAL\%20PAPER.pdf.

[22] Hackworth N. Hamilton V. Moore S Northam E. Bucalo Z Cameron F. Predictors of Diabetes Self-care, Metabolic Control, and Mental Health in Youth with Type 1 Diabetes. Australian Psychologist 48 (2013) 360-368.

[23] Vasli P. Eshghbaz F. Evaluation of selfcare children with diabetes type I and its association with family reaction. J Mazandaran Univ Med Sci. 2009; 19(69): 38-44. 
[24] Salehi S. Shafiei S. Amini P. Abdeyazdan GH. Self-care performance in children and adolescents with type 1 diabetes mellitus. Jundishapur J Chronic Dis Care 2014; 3(3): e21706.

[25] Attia A. Hassan E. Effect of instructions on selected self-care practices among type-1 diabetic children. Egyptian nursing journal 2017; 14 (2): 179-89.

[26] Greene M, Mandleco,B, Roper S, Marshall E, Dyches T. Metabolic control, self-care behaviors, and parenting in adolescents with type 1 diabetes. SAGE journals 2010; 36 (2): 326-336.

[27] Formosa N. Blood glucose monitoring in children and adolescents with type 1 diabetes mellitus. MMJ 2013; 25: 31-5.

[28] Tanjia N. Fannana T. Naved M. Sultana S. Assessing the Knowledge, Attitude and Practice of Diabetes Mellitus among Diabetes Patients in Dhaka City, Bangladesh. J Pharm Chem Biol Sci , 2016; 4(1): 64-75.

[29] Santiprabhob J, Kiattisakthavee P, Likitmaskul S, Chaichanwattanakul K, Wekawanich J, Dumrongphol $H$, Sriwijitkamol A, Peerapatdit T, Nitiyanant W. Glycemic control, quality of life and self-care behavior among adolescents with type 1 diabetes who attended a diabetes camp. Southeast Asian J Trop Med Public Health 2012; 43 (1): 172-84.

[30] Naguib JM, Kulinskaya E, Lomax CL, et al. Neuro-cognitive performance in children with type 1 diabetes-a meta-analysis. $J$ Pediatr Psychol 2009; 34:271-82.

[31] Welch G, Garb J, Zagarins S, Lendel I, Gabbay RA. Nurse diabetes case management interventions and blood glucose control: results of a meta-analysis. Diabetes Res Clin Pract 2010; 88(1): 1-6.

[32] Silva A, Apolonio M, De Alcantara C, Queiroz M. Sociocultural characteristics of adolescents with type 1 diabetes mellitus. Cogitare enferm. $2016 ; 21(4): 1-7$.

[33] Shrivastava SR, Shrivastava PS, Ramasamy J. Role of self-care in management of diabetes mellitus. Journal of Diabetes and Metabolic Disorders. 2013; 12: 14.

[34] Nam S, Chesla C, Stotts N, Kroon L, Janson S. Barriers to diabetes management: patient and provider factors. Diabetes Res Clin Pract 2011; 93(1): 1-9.

[35] Desalu O, Salawu F, Jimoh A, Adekoya A, Busari O, Olokoba A. Diabetic foot care: self-reported knowledge and practice among patients attending three tertiary hospital in Nigeria. Ghana Med $\mathrm{J}$ 2011; 45: 60-5.
[36] Stetson B, Boren S, Leventhal H. Embracing the evidence on problem solving in diabetes self-management education and support. Self-Care 2010; 1(3): 83-116.

[37] Gomes M. Santos D. Pizarro M. Barros B. de Melo L. Negrato C. Does knowledge on diabetes management influence glycemic control? A nationwide study in patients with type 1 diabetes in Brazil. Patient Preference and Adherence 2018:12:53 62

[38] Ryan M. An assessment of knowledge and self-care practices in patients living with type 2 diabetes mellitus. Published master thesis, University of Malta; Faculty of Health Sciences, department of Podiatry [Internet] 2014 [Cited August 17]. Available from https://www.um.edu.mt/library/oar//handle/123456789/9994.

[39] Soheilipour F. Jolfaei A. Khodapanahandeh F. Rajab A. Salehiniya H. Asoudegi M. Tamannaie Z. Rahimzadeh N. The relationship between maternal awareness, socioeconomic situation of families and metabolic control in children with type 1 diabetes in an Iranian population. J Compr Ped. 2015; 6(3): e26924.

[40] Niba LL, Aulinger B, Mbacham WF, Parhofer KG. Predictors of glucose control in children and adolescents with type 1 diabetes: results of a cross-sectional study in Cameroon. BMC Research Notes. 2017; 10: 207.

[41] Basu S, Khobragade M, Raut DK, Garg S. Knowledge of diabetes among diabetic patients in government hospitals of Delhi. International journal of noncommuicable diseases 2017; 2(1): 8-10.

[42] Onuoha PH. Knowledge and Practice of self-care Management of Persons with Type 11 Diabetes at a Health Centre in East Trinidad. International Journal of Development Research 2017; 7(9): 15530-9

[43] Rezaeian M, Sharifirad G, Mostafavi F, Moodi M, Abbasi MH. The effects of $\mathrm{BC}$ educational intervention on knowledge and health beliefs of women 40 years and older, Isfahan, Iran. Journal of Education and Health Promotion 2014;3 (43):59-64.

[44] Flora M and Gameiro M. Self-care of adolescents with type 1 diabetes: responsibility for disease management. Journal of Nursing Referência 2016; 9 (1): 9-19.

[45] Moskovitz A, Knee A, Frydman E, Allen H, Tonyushkina K. Diabetes Knowledge in Youth with T1DM and their Caregivers-A Cross-Sectional Study. J Diabetes Clin Endocrinol 2018; 1(1): 101. 